\title{
MicroRNA-486-5p functions as a tumor suppressor of proliferation and cancer stem-like cell properties by targeting Sirt1 in liver cancer
}

\author{
XINLONG YAN ${ }^{1-3^{*}}$, XINHUI LIU ${ }^{1-3 *}$, ZHAOHAI WANG ${ }^{4 *}$, QIAN CHENG ${ }^{5^{*}}$, GUANGHONG JI $^{2,3}$, \\ HUI YANG ${ }^{1-3}$, LINGFEI WAN ${ }^{1-3}$, CHEN GE ${ }^{1-3}$, QUAN ZENG ${ }^{2,3}$, HUA HUANG ${ }^{1}$, \\ JIAFEI XI ${ }^{2,3}$, LIJUAN HE ${ }^{2,3}$, XUE NAN ${ }^{2,3}$, WEN YUE ${ }^{2,3}$ and XUETAO PEI ${ }^{2,3}$ \\ ${ }^{1}$ College of Life Science and Bioengineering, Beijing University of Technology, Beijing 100124; \\ ${ }^{2}$ Stem Cell and Regenerative Medicine Laboratory, Institute of Health Service and Transfusion Medicine, \\ Beijing 100850; ${ }^{3}$ South China Institute of Biomedicine, Guangzhou, Guangdong 510005; \\ ${ }^{4}$ Department of Gastroenterology and Hepatology, Beijing 302 Hospital, Beijing 100039; \\ ${ }^{5}$ Department of Hepatobiliary Surgery, Peking University People's Hospital, Beijing 100044, P.R. China
}

Received May 3, 2018; Accepted November 28, 2018

DOI: $10.3892 / o r .2018 .6930$

\begin{abstract}
Cancer stem-like cells (CSCs) are critical for the initiation, progression, chemoresistance and postsurgical recurrence of liver cancer. They are thought to be novel targets for the treatment of liver cancer, however, efficient agents that target liver cancer stem cells (CSCs) have not been identified. MicroRNAs (miRNAs) are small non-coding RNAs that target the 3'untranslated region (3'UTR) of mRNAs. Their dysregulation has been implicated in several types of cancer including liver cancer, but it still remains unknown if they play a role in targeting liver CSCs. We compared the miRNA profiles between liver cancer samples and adjacent non-tumor tissues using The Cancer Genome Atlas (TCGA) datasets. Several miRNAs including miR-486-5p (miR-486) were found to be significantly downregulated in liver cancer tissues. These differentially expressed miRNAs were screened between CSC-enriched tumor spheres and adherent cells. miR-486 was significantly downregulated in tumor spheres and liver cancer samples. Ectopic expression of miR-486 significantly repressed the self-renewal and invasion of CSCs in vitro and tumorigenesis in vivo. Notably, we found that sirtuin 1 (Sirt1) served as a direct target of miR-486. The high expression of Sirt1 was involved
\end{abstract}

Correspondence to: Professor Wen Yue or Professor Xuetao Pei, Stem Cell and Regenerative Medicine Laboratory, Institute of Health Service and Transfusion Medicine, 27 Taiping Road, Haidian, Beijing 100850, P.R. China

E-mail: wenyue226@126.com

E-mail:peixt@nic.bmi.ac.cn

*Contributed equally

Key words: liver cancer, miR-486, cancer stem cells, Sirt1 in maintaining the self-renewal and tumorigenic potential of liver CSCs. The results of the present study indicated that the miR-486-Sirt1 axis was involved in suppressing CSC traits and tumor progression.

\section{Introduction}

Liver cancer is a common and devastating malignancy with high mortality worldwide (1). High-throughput sequencing of liver cancer tissues has revealed that liver cancer samples possess extensive intra-tumor heterogeneity $(2,3)$. Most types of cancers contain a small population of highly tumorigenic and drug-resistant cells known as cancer stem cells (CSCs) or tumor initiating cells (TICs) $(4,5)$. These cells have the capacity to self-renew, differentiate, and generate tumors identical to the original one in primary and metastatic sites (6). CSCs are thought to be responsible for tumor initiation, progression, chemoresistance and postsurgical recurrence of liver cancer patients (7). Therefore, therapies that specifically target liver CSCs may be beneficial for the treatment of liver cancer.

Diverse surface markers such as cluster of differentiation 133 (CD133) (8,9), CD90 (10), epithelial cellular adhesion molecule (EpCAM) (11), CD13 (12,13), cytokeratin 19 (CK19) (14) and oval cell marker (OV-6) (15), or side population cells that efflux the DNA-binding dye Hoechst 33342 have been used to identify liver CSCs $(16,17)$. However, due to the low percentage of CSCs existing in tumor patients and cell lines, and the fact that cell surface antigens are disrupted by enzymatic digestion, the isolation efficiency of CSCs is very low. Therefore, an easy-to-use method of tumor sphere formation has been increasingly used to enrich CSC-like cells in many cancers including liver cancer (18), since tumor sphere-forming cells from tumor patients and cell lines possess CSC-like features and tumorigenic potential. In the present study, we used this method to enrich liver CSCs and to identify differentially expressed miRNAs between CSC-enriched tumor spheres and adherent cells. 
MicroRNAs (miRNAs) are a class of small non-coding RNAs ( 22 nucleotides) that repress gene expression at the post-transcriptional level by binding to the 3'UTR of target genes $(19,20)$. Several miRNAs have been implicated in liver cancer proliferation, metastasis, chemoresistance $(21,22)$, and interactions between the tumor microenvironment and liver cancer cells (23). For example, miRNA-125b, which is downregulated in liver cancer cells, significantly suppresses epithelial-mesenchymal transition (EMT) associated traits in liver cancer cells targeting SMAD2 and SMAD4 (24). A strategy of restoring tumor-repressing miRNA expression in liver cancer cells may be used to inhibit the proliferation, metastasis and self-renewal of liver CSCs.

In the present study, we enriched liver CSCs using tumor spheres or two surface markers, CD13 and EpCAM. Then, we screened for significantly downregulated miRNAs from The Cancer Genome Atlas (TCGA) database in CSCs and adherent cells. Among them, miR-486 was found to be significantly downregulated in tumor spheres and liver cancer tissues. Overexpression of miR-486 suppressed tumor sphere expansion, and the invasion and tumorigenicity of liver cancer cells. The miRecords and RNAhybrid algorithms predicted that Sirtl was a direct target of miR-486. Luciferase reporter assays and immunohistochemistry (IHC) staining indicated that miR-486 significantly reduced Sirt1 expression. We also found that Sirt1 was responsible for maintaining the self-renewal and tumorigenicity of liver CSCs. The results of the present study suggest that increasing miR-486 levels in liver cancer or liver CSCs may be a promising strategy for liver cancer treatment.

\section{Materials and methods}

Patient specimens. Liver cancer tissues and corresponding non-tumor tissues were obtained from the Beijing 302 Hospital. Sixteen HCC samples (14 males and 2 females; range, 49-65 years) were obtained from consecutive patients undergoing initial hepatectomy from January 2015 to December 2017. All human specimens were collected in accordance with the Declaration of Helsinki, and the protocols involving clinical samples were approved by the Research Ethics Committees of theAcademy of Military Medical Sciences, Beijing, China. Written informed consent was obtained from all patients.

Cell culture. Liver cancer cells including Huh7, Hep3B, Li-7 and PLC/PRF/5, (CRL-8024 ${ }^{\mathrm{TM}}$; also referred to as PLC) from the American Type Culture Collection (ATCC; Manassas, VA, USA), the MHCC97H (briefly, 97H), MHCC97L (briefly, 97L), HCCLM3 (briefly, LM3) cells were obtained from the Liver Cancer Institute of Fudan University (Shanghai, China) (25). The HepG2 cells were obtained from the National Platform for Experimental Cell Resources (Beijing, China). These cells were maintained in Dulbecco's modified Eagle's medium (DMEM) supplemented with $10 \%$ fetal bovine serum (FBS), $100 \mathrm{U} / \mathrm{ml}$ penicillin and $100 \mathrm{ng} / \mathrm{ml}$ streptomycin at $37^{\circ} \mathrm{C}$ under $5 \% \mathrm{CO}_{2}$.

TCGA database. The TCGA database (https://tcga-data.nci. nih.gov/tcga/) was used to analyze differentially expressed miRNAs between liver cancer and adjacent non-tumor tissues.
Quantitative real-time PCR ( $q P C R)$. Total RNAs from liver cancer specimens and cells were extracted using TRIzol (Invitrogen; Thermo Fisher Scientific, Inc., Waltham, MA, USA). Reverse transcription of total RNA into cDNA was performed with the miScript Reverse Transcription kit (Qiagen Sciences, Inc., Gaithersburg, MD, USA), and qPCR was performed using the miScript SYBR-Green PCR Master Mix on the ABI Prism 7900 system (Applied Biosystems; Thermo Fisher Scientific, Inc.). Briefly, a $20 \mu 1 \mathrm{RTqPCR}$ system was performed for 40 cycles according to the following conditions: An initial denaturation was performed at $95^{\circ} \mathrm{C}$ for $3 \mathrm{~min}$, followed by denaturation at $95^{\circ} \mathrm{C}$ for $15 \mathrm{sec}$, annealing at $58^{\circ} \mathrm{C}$ for $30 \mathrm{sec}$, and extension at $72^{\circ} \mathrm{C}$ for $7 \mathrm{~min}$. Relative quantification of miRNA or mRNA expression was calculated using the $2^{-\triangle \Delta C q}$ method (26). 18S was used as an mRNA internal control. U6 RNA was used as a miRNA internal control. Each experiment was conducted with at least three independent replicates. Primer sequences are listed in Table I.

Flow cytometry and sorting. Fluorescence-activated cell sorting was performed to enrich $\mathrm{EpCAM}^{+}$and $\mathrm{CD}_{13}{ }^{+}$liver cancer cells. Briefly, Huh7 cells were trypsinized and washed twice with phosphate-buffered saline (PBS), then incubated with APC-anti-human EpCAM (dilution 1:200; cat. no. 324208; BioLegend, San Diego, CA, USA) or APC-anti-human CD13 antibody (dilution 1:100; cat. no. 301706; BioLegend) for $30 \mathrm{~min}$ at $4^{\circ} \mathrm{C}$. After incubation, Huh7 cells were washed 3 times and then sorted on the FACSAria II instrument (BD Biosciences, San Jose, CA, USA). Huh7-EpCAM ${ }^{+}$(or CD13 ${ }^{+}$) and Huh7-EpCAM- (or CD13 ${ }^{-}$) cells were collected for qPCR analysis.

Tumor sphere culture. A total of 1,000 tumor cells were seeded in the 6-well ultra-low attachment plates (Corning Inc., Corning, NY, USA) and cultured in DMEM/F-12 supplemented with B27, N2 (both from Invitrogen; Thermo Fisher Scientific, Inc.), $10 \mathrm{ng} / \mathrm{ml}$ of epidermal growth factor (EGF), $5 \mathrm{ng} / \mathrm{ml}$ of basic fibroblast growth factor (bFGF), $100 \mathrm{U} / \mathrm{ml}$ penicillin and $100 \mathrm{ng} / \mathrm{ml}$ streptomycin at $37^{\circ} \mathrm{C}$ under $5 \% \mathrm{CO}_{2}$ for 7-10 days as we previously described $(27,28)$.

Oligonucleotides. Transient expression of the miR-486 mimics or miR-486 inhibitors have been previously described (29). Scramble miRNA, lenti-miR-486 and lenti-miR-486 inhibitor were purchased from Suzhou GenePharma Co., Ltd. (Suzhou, China). The sequence of hsa-miR-486 mimics was: TCCTGT ACTGAGCTGCCCCGAG, that of hsa-miR-486 inhibitor was: ACCCCTATCACGATTAGCATTAA, and that of the miRNA inhibitor negative control (NC) was: CAGTACTTT TGTGTAGTACAA.

Short hairpin RNA (shRNA) and lentivirus package. To generate lentivirus plasmids for stable RNA interference, short hairpins were designed using online software (http://rnaidesigner.lifetechnologies.com/rnaiexpress/design. do) as previously described (30). Based on the Sirt1 sequence, the small interfering RNA (siRNAs) Sirt1 sequence was 5'-GGUGCCGUGCUACUCAUAUTT-3'. The Sirt1-specific short hairpin (shRNA) expression vector and the scrambled ineffective shRNA cassette were cloned into the pSicoR-GFP 
Table I. The sequences of qRT-PCR primers in the study.

\begin{tabular}{|c|c|c|}
\hline Gene/miRNA & Forward primer $\left(5^{\prime}-3^{\prime}\right)$ & Reverse primer $\left(5^{\prime}-3^{\prime}\right)$ \\
\hline miRNA universal primer & GATTGAATCGAGCACCAGTTAC & \\
\hline U6 & CGCTTCGGCAGCACATATACTA & miRNA universal primer \\
\hline $\operatorname{miR}-486-5 p$ & TCCTGTACTGAGCTGCCCCGAG & miRNA universal primer \\
\hline miR-214 & ACAGCAGGCACAGACAGGCAGT & miRNA universal primer \\
\hline $\operatorname{miR}-187$ & TCGTGTCTTGTGTTGCAGCCGG & miRNA universal primer \\
\hline miR-511-3p & AATGTGTAGCAAAAGACAGA & miRNA universal primer \\
\hline miR-101 & TACAGTACTGTGATAACTGAA & miRNA universal primer \\
\hline miR-99a & AACCCGTAGATCCGATCTTGTG & miRNA universal primer \\
\hline miR10a & TACCCTGTAGATCCGAATTTGTG & miRNA universal primer \\
\hline miR-337 & СТCCTATATGATGCCTTTCTTC & miRNA universal primer \\
\hline $\operatorname{miR}-199 a-3 p$ & ACAGTAGTCTGCACATTGGTTA & miRNA universal primer \\
\hline $\operatorname{miR}-483$ & TCACTCCTCTCCTCCCGTCTT; & miRNA universal primer \\
\hline $\operatorname{miR}-33 b$ & GTGCATTGCTGTTGCATTGC & miRNA universal primer \\
\hline $\operatorname{miR}-490$ & CAACCTGGAGGACTCCATGCTG & miRNA universal primer \\
\hline $\operatorname{miR}-451$ & AAACCGTTACCATTACTGAGTT & miRNA universal primer \\
\hline $\operatorname{miR}-138$ & AGCTGGTGTTGTGAATCAGGCCG & miRNA universal primer \\
\hline $\operatorname{miR}-223$ & TGTCAGTTTGTCAAATACCCCA & miRNA universal primer \\
\hline $\operatorname{miR}-135 b$ & TATGGCTTTTCATTCCTATGTGA & miRNA universal primer \\
\hline $\operatorname{miR}-195$ & TAGCAGCACAGAAATATTGGC & miRNA universal primer \\
\hline $\operatorname{miR}-375$ & TTTGTTCGTTCGGCTCGCGTGA & miRNA universal primer \\
\hline miR-139 & TCTACAGTGCACGTGTCTCCAGT & miRNA universal primer \\
\hline $\operatorname{miR}-1258$ & AGTTAGGATTAGGTCGTGGAA & miRNA universal primer \\
\hline $\operatorname{miR}-145$ & GTCCAGTTTTCCCAGGAATCCCT & miRNA universal primer \\
\hline $\operatorname{miR}-199 a-5 p$ & CCCAGTGTTCAGACTACCTGTTC & miRNA universal primer \\
\hline $\operatorname{miR}-383-5 p$ & AGATCAGAAGGTGATTGTGGCT & miRNA universal primer \\
\hline $\operatorname{miR}-411$ & TAGTAGACCGTATAGCGTACG & miRNA universal primer \\
\hline miR-592 & TTGTGTCAATATGCGATGATGT & miRNA universal primer \\
\hline miR-144 & TACAGTATAGATGATGTACT & miRNA universal primer \\
\hline $\operatorname{miR}-450$ & TTTTGCGATGTGTTCCTAATAT & miRNA universal primer \\
\hline $\operatorname{miR}-424$ & CAGCAGCAATTCATGTTTTGAA & miRNA universal primer \\
\hline $\operatorname{miR}-326$ & СCTCTGGGCCCTTCCTCCAG & miRNA universal primer \\
\hline $\operatorname{miR}-150$ & TCTCCCAACCCTTGTACCAGTG & miRNA universal primer \\
\hline miR-379 & TGGTAGACTATGGAACGTAGG & miRNA universal primer \\
\hline miR-154 & TAGGTTATCCGTGTTGCCTTCG & miRNA universal primer \\
\hline $\operatorname{miR}-497$ & CAGCAGCACACTGTGGTTTGT & miRNA universal primer \\
\hline miR-204 & TTCCCTTTGTCATCCTATGCCT & miRNA universal primer \\
\hline $\operatorname{miR}-378$ & CTCCTGACTCCAGGTCCTGTGT & miRNA universal primer \\
\hline $18 \mathrm{~S}$ & AACCCGTTGAACCCCATT & CCATCCAATCGGTAGTAGCG \\
\hline Sox 2 & GCCGAGTGGAAACTTTTGTCG & GGCAGCGTGTACTTATCCTTCT \\
\hline OCT4 & GGGAGATTGATAACTGGTGTGTT & GTGTATATCCCAGGGTGATCCTC \\
\hline CD13 & TTCAACATCACGCTTATCCACC & AGTCGAACTCACTGACAATGAAG \\
\hline Sirt1 & TAGCCTTGTCAGATAAGGAAGGA & ACAGCTTCACAGTCAACTTTGT \\
\hline TNC & GCCCCTGATGTTAAGGAGCTG & GGCCTCGAAGGTGACAGTT \\
\hline ANXA13 & GCTAAAGCGAGCAGTCCTCAG & GTCCTGCCCGATAAGATTTCAA \\
\hline
\end{tabular}

plasmid. Virus packaging was performed in 293T cells after co-transfection of lentiviral expression plasmids with the packaging plasmids (pRRE, pCMV-VSVG and pRSV-REV; Addgene, Inc., Cambridge, MA, USA) using Lipofectamine 2000. The viral supernatant was used to infect liver cancer cells, and stable GFP-expressing clones were selected by fluorescence-activated cell sorting (FACS).

Luciferase reporter assay. The 3'UTR sequences of Sirt1 that contained the predicted complimentary sites of miR-486 
A TCGA: downregulated miRNAs in HCC vs. Adjacent non-tumor tissues
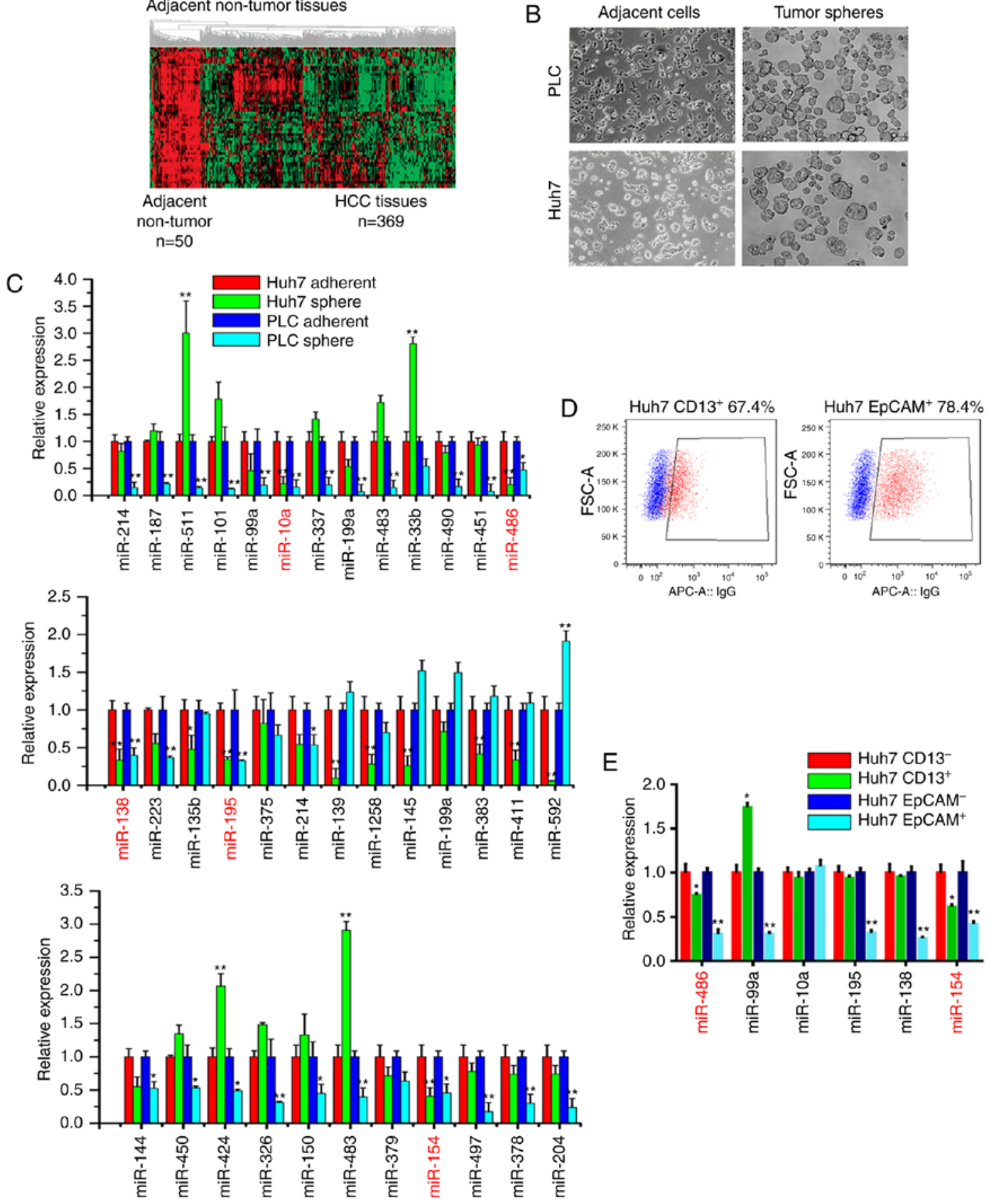

Figure 1. miR-486 is significantly downregulated in CSC-like cells. (A) The TCGA database indicated the differential miRNA expression in liver cancer $(n=369)$ and adjacent non-tumor tissues $(n=50)$. (B) Morphology of liver tumor spheres and paired adherent cells. (C) Comparison of selected miRNA expression levels in tumor spheres and adherent cells. (D) Isolation of CD13 and EpCAM positive and negative populations in Huh7 cells by FACS assays. (E) Differential miRNA expression levels were further confirmed in $\mathrm{CD}^{+} 3^{+}$and $\mathrm{EpCAM}^{+}$cells by qPCR assays. ${ }^{*} \mathrm{P}<0.05,{ }^{* *} \mathrm{P}<0.01$. CSC, cancer stem cells; EpCAM, epithelial cellular adhesion molecule.

were cloned into the pGL3 basic reporter vector (Promega Corp., Madison, WI, USA). The pGL3-Sirt1-3'UTR vector or pGL3 basic vector was co-transfected with miR-486 mimics or NC into $293 \mathrm{~T}$ cells, with the Renilla luciferase vector used as an internal control. After $48 \mathrm{~h}$, cells were harvested, and the luciferase activity was detected using the Dual-Luciferase assay kit (Promega). The ratio of firefly/Renilla luciferase activities was calculated and designated as the relative promoter activity.
Cell invasion assays. Cell invasion assays were performed in Transwell chambers coated with Matrigel ( $8 \mu \mathrm{m}$ Transwell inserts; BD Biosciences). A total of $1 \times 10^{6}$ cells in serum-free DMEM were seeded in the upper chamber, and DMEM with $5 \%$ FBS was added to the bottom chamber as an attractant. After $48 \mathrm{~h}$ of incubation, the penetrated cells on the filters were fixed in $20 \%$ methanol and stained with crystal violet. Ten randomly selected fields (magnification, x100) in each well were counted under a light microscope. 


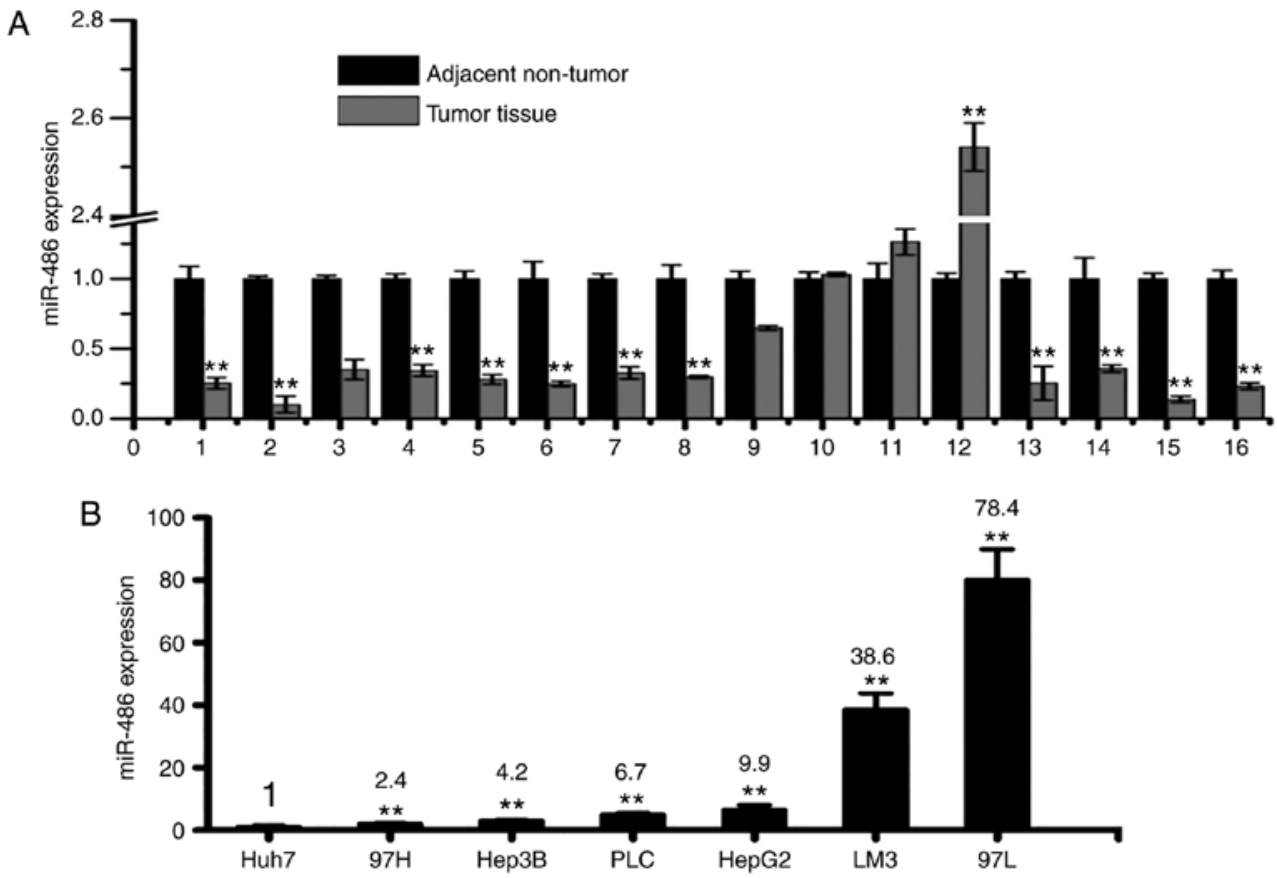

Figure 2. miR-486 expression in liver cancer tissues and cell lines. (A) qPCR analysis of miR-486 levels in paired liver cancer and adjacent non-tumor tissues, $\mathrm{n}=16$. (B) The miR-486 expression in liver cancer cell lines was determined by qPCR assays. ${ }^{* *} \mathrm{P}<0.01$.

Scratch healing assays. The effects of miR-486 on the migration of tumor cells were determined by wound healing assays. A total of $5 \times 10^{5}$ cells/well were seeded and cultured in 6-well plates to create a confluent monolayer. After the cells attached overnight, a straight line was scraped with a $200-\mu 1$ pipette tip across the cell monolayer to create a 'scratch'. After $48 \mathrm{~h}$, microscopic images of the 'scratch closure' were captured under a light microscope at an original magnification of $x 4$.

In vivo tumorigenicity assays. Female nude mice (6-8 weeks old) were purchased from Weitonglihua Company (Beijing WeitongLihua Experimental Animal Technology Co., Ltd., Beijing, China) and raised under specific pathogen-free conditions. Mice were allowed ad libitum access to food and water and were maintained on a constant light dark cycle with constant temperature and humidity. All animal experiments in the present study were approved by the Ethics Committee of the Academy of Military Medical Sciences. Huh7-miR-486 or HepG2-miR-486 and corresponding control cells $\left(2 \times 10^{6}\right)$ were subcutaneously injected into the right and left side of mice, respectively. Similarly, Hep3B-shSirt1 or HepG2-shSirt1 and control cells were subcutaneously injected into nude mice. Approximately 5 weeks after injection, mice were anesthetized with $1 \%$ pentobarbital sodium $(75 \mathrm{mg} / \mathrm{kg})$ through the intraperitoneal (IP) administration, and sacrificed by cervical dislocation, and the tumors were weighed as previously described (23). Non-retrospective ethical approval was obtained for the animal experiments conducted in the study. Tumor burden did not exceed the recommended dimensions.

Statistical analysis. The data are presented as the means \pm standard deviation (SD) from at least 3 independent experiments. Quantitative results were compared using
GraphPad Prism version 5.0 software (GraphPad Software, Inc., La Jolla, CA, USA). A two-tailed Student's paired t-test was used to test for significance between two groups. Multiple groups were compared by a one- or two-way analysis of variance with Tukey's post hoc correction. Statistical significance was regarded as $\mathrm{P}<0.05$ or $\mathrm{P}<0.01$.

\section{Results}

miR-486 is preferentially downregulated in liver CSC-like cells and liver cancer tissues. To identify differentially expressed miRNAs between CSC-like cells and adherent cells, we first used the TCGA miRNA sequence database to analyze the altered miRNAs between liver cancer specimens $(n=369)$ and adjacent non-tumor tissues $(\mathrm{n}=50)$. We mainly focused on the downregulated miRNAs in liver cancer tissues, and identified a set of 59 miRNAs that were significantly downregulated in liver cancer specimens compared with adjacent non-tumor tissues, including miR-486, miR-99a, miR-195 and miR-154 (Fig. 1A). Then, we enriched liver CSC-like cells using tumor sphere models (Fig. 1B), and qPCR assays were further utilized to compare the differentially expressed miRNAs between tumor spheres and adherent cells. As shown in Fig. 1C, the expression levels of miR-99a, miR-10a, miR-486, miR-195, miR-154 and miR-138 in liver tumor spheres were significantly decreased compared with adherent cells. Moreover, liver CSCs were enriched with CSC surface markers including EpCAM and CD13. Thus, we performed fluorescence-activated cell sorting (FACS) to separate Huh7 cells into $\mathrm{CD}^{-} 3^{-}$and $\mathrm{CD}^{+} 3^{+}$, and $\mathrm{EpCAM}^{-}$and $\mathrm{EpCAM}^{+}$ subsets, respectively (Fig. 1D). According to the qPCR results, miR-486 was significantly downregulated in $\mathrm{EpCAM}^{+}$and $\mathrm{CD}^{+} 3^{+}$subpopulations (Fig. 1E). Moreover, miR-486 levels were significantly decreased in tumor samples compared with 
A
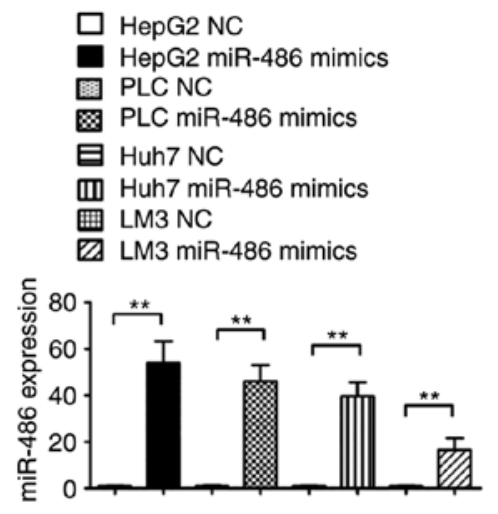

B

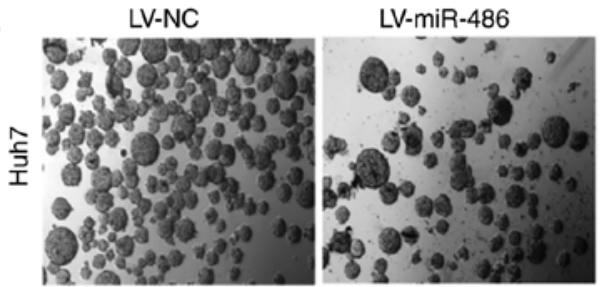

$\square$ HepG2 NC inhibitor

HepG2 miR-486 inhibitor

PLC NC inhibitor

ख PLC miR-486 inhibitor

目 Huh7 NC inhibitor

血 Huh7 miR-486 inhibitor

四 LM3 NC inhibitor

Z LM3 miR-486 inhibitor
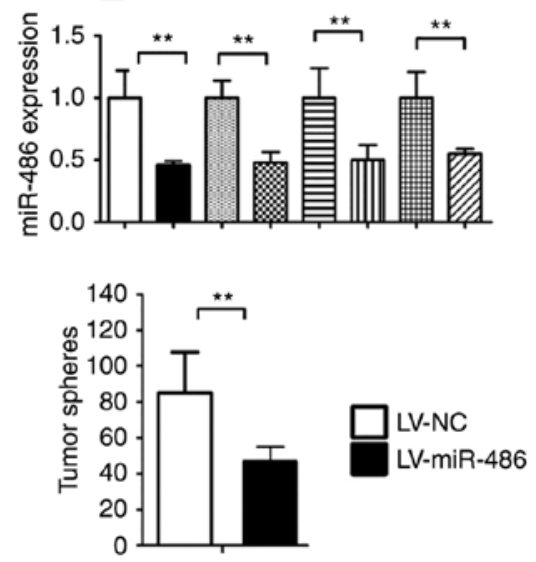

$\square$ Huh7 NC inhibitor

- Huh7 miR-486 inhibitor

$\square$ LM3 NC inhibitor

뜸 LM3 miR-486 inhibitor

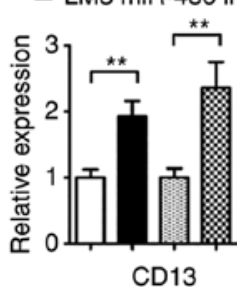

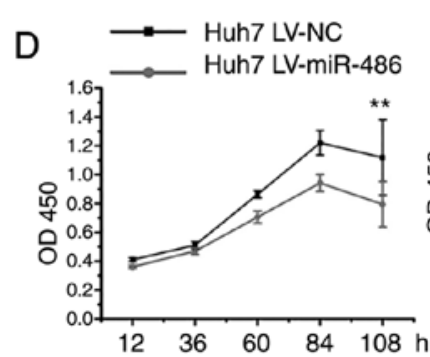

\section{$\longrightarrow$ LM3 LV-NC}
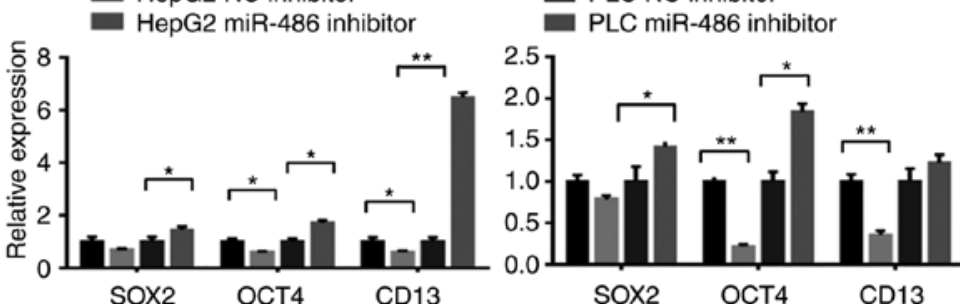

PLC NC
PLC miR-486 mimics

PLC NC inhibitor

- PLC miR-486 inhibitor

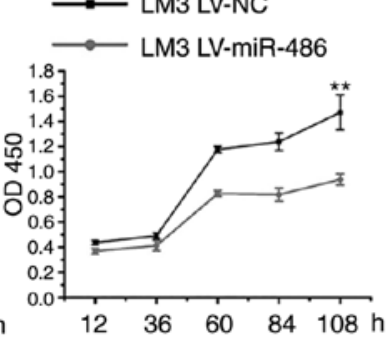

$E$

Huh7 LV-NC Huh7 LV-miR-486

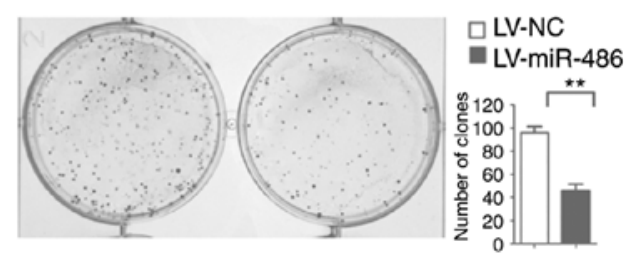

$\mathrm{F}$
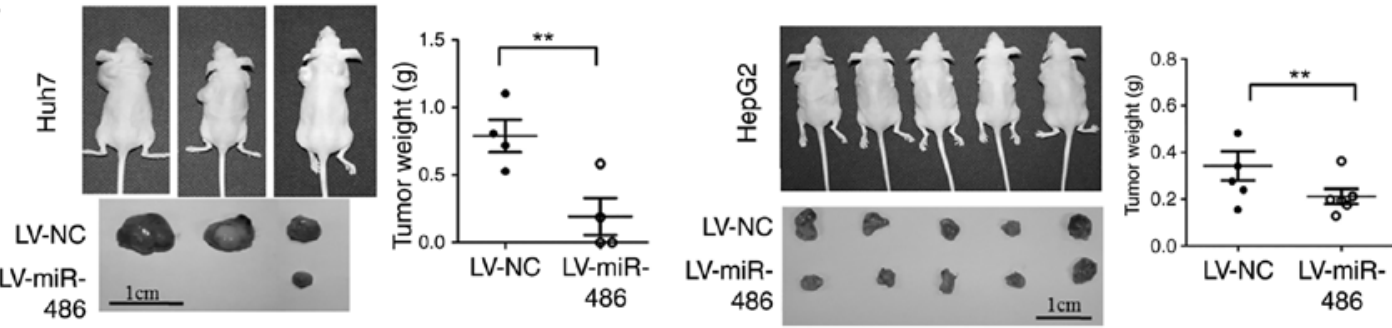

Figure 3. miR-486 markedly suppresses tumor stemness properties of liver cancer cells. (A) The relative expression of miR-486 in miR-486 mimics- (overexpression) or miR-486 inhibitor-transfected liver cancer cells (HepG2, PLC, Huh7 and LM3). (B) miR-486 overexpression significantly suppressed tumor sphere formation in Huh7 cells. (C) CSC-related genes including SOX2, OCT4 and CD13 were analyzed in liver cancer cells transfected with miR-486 mimics or miR-486 inhibitors. CD13 expression was further confirmed in two other tumor cell lines (Huh7 and LM3) when transfected with miR-486 inhibitor. (D) CCK-8 assays and (E) clone forming capacity analysis were used to compare the proliferation capacity of miR-486 overexpression in liver cancer cells in vitro. (F) Liver cancer cells $\left(2 \times 10^{6}\right)$ were subcutaneously injected into nude mice. NC mimic-transfected cells were injected in the left side and the miR-486-overexpressed cells were injected in the right side. After 6 weeks, images of the mice were captured, and the tumor mass was weighed. Data are presented as the means $\pm \mathrm{SD}$. ${ }^{*} \mathrm{P}<0.05,{ }^{* * *} \mathrm{P}<0.01$. CSC, cancer stem cells; CCK-8, Cell Counting Kit-8.

corresponding non-tumor tissues (Fig. 2A) and most tumor cell lines (Fig. 2B). Among the 10 most frequently used liver cancer cell lines, miR-486 was lowly expressed in Huh7 (highly proliferative) $(23,29)$ and $97 \mathrm{H}$ cells (highly invasive) $(25,31)$. 
A

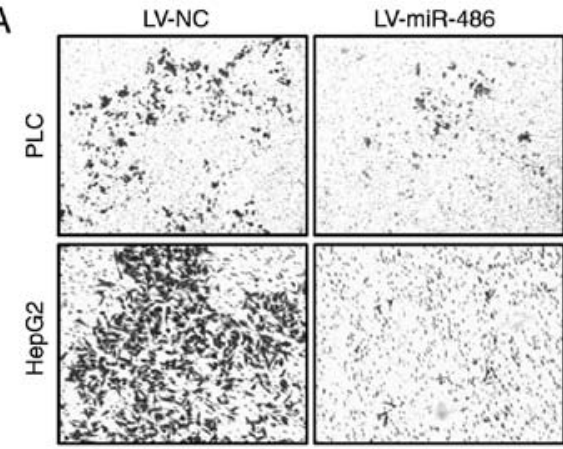

口LV-NC

LV-miR-486

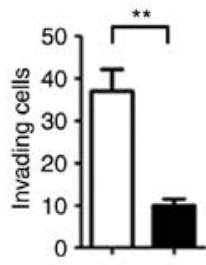

B

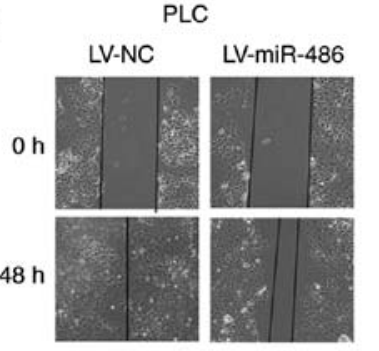

$\square$ LV-NC

LV-miR-486

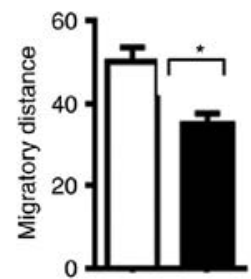

HepG2

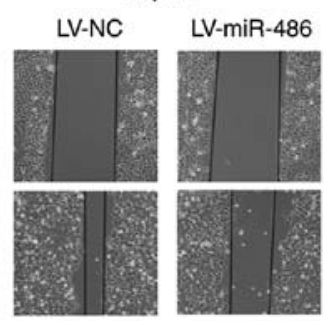

LV-NC

- LV-miR-486

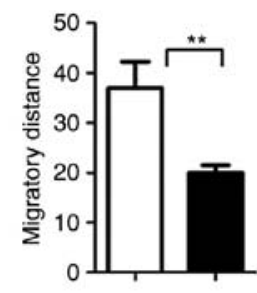

Figure 4. Involvement of miR-486 in the invasion and migration of liver cancer cells. (A) miR-486 overexpression significantly suppressed the invasion abilities of liver cancer cells. (B) The migratory capacity of tumor cells was markedly reduced by miR-486 transfection. Data are displayed as the means \pm SD from at least 3 independent experiments. ${ }^{*} \mathrm{P}<0.05,{ }^{* *} \mathrm{P}<0.01$.

In our laboratory, only 3 liver cancer cell lines including Huh7, PLC/PRF/5 and Li-7 cells could form tumor spheres easily in vitro (data not shown). The tumor sphere-forming cells from tumor patients and cell lines possessed more CSC-like features and tumorigenic potential. In addition, the Huh7 cells could easily form tumors in vivo than PLC/PRF/5 and Li-7 cells (data not shown). These results indicated that miR-486 was significantly downregulated in liver CSCs and liver cancer tissues.

miR-486 significantly suppresses the tumor stemness and invasion of liver cancer cells. First, we confirmed the relative expression of miR-486 in miR-486 overexpression and inhibitor expression in liver cancer cells (Fig. 3A). Compared to the $\mathrm{NC}$ group, the number of tumor spheres was decreased in Huh7 cells transfected with lentivirus miR-486 (LV-miR-486) (Fig. 3B). miR-486 overexpression suppressed the expression of stemness-related gene CD13. Conversely, miR-486 inhibitor promoted the expression of CD13 in liver cancer cells (Fig. 3C). The Cell Counting Kit-8 (CCK-8) assays indicated that miR-486 overexpression significantly suppressed the proliferation of Huh7 and LM3 cells in vitro (Fig. 3D). Moreover, the clone-forming capacity in miR-486 overexpression Huh7 cells was significantly decreased compared with NC group (Fig. 3E). Then, Huh7-miR-486 and Huh7-NC cells were subcutaneously injected into nude mice. Consequently, the tumor sizes and weights in the Huh7-miR-486 group were significantly decreased compared to the Huh7-NC group $(\mathrm{P}<0.01$; Fig. 3F, left panel). Similar results were observed in mice injected with HepG2-miR-486 cells $(\mathrm{P}<0.01)$ (Fig. 3F, right panel). The cell invasion and scratch healing assays revealed that miR-486 mimics significantly decreased the number of invading cells (Fig. 4A) and the migration distance of liver cancer cells (Fig. 4B). Collectively, these results demonstrated that miR-486 has tumor suppressive functions in CSC traits.

Sirtl is a direct target of miR-486 in liver CSC cells. miRNAs usually bind to the complementary sites in the 3'UTRs of target mRNAs and trigger RNA degradation or translation repression (32). Both TargetScan and miRanda database predicted that Annexin A13 (ANXA13), Sirt1 and tenascin-C (TNC) could serve as potential target genes of miR-486 (Fig. 5A). The results of the qPCR analysis confirmed that miR-486 suppressed the expression of Sirt1 and TNC in HepG 2 and PLC cells. Conversely, Sirt1 and TNC mRNA levels were increased with transfection of a miR-486 inhibitor (Fig. 5B). Our preliminary results indicated that knockdown of TNC did not inhibit the growth of tumor spheres in vitro (data not shown). Thus, in the present study, we mainly focused on Sirtl as the target gene of miR-486. To further elucidate the interaction between miR-486 and Sirt1 3'UTR, a luciferase assay was subsequently performed in 293T cells. The pGL3-Sirt1-3'UTR vector or pGL3 basic vector was co-transfected with miR-486 mimics or $\mathrm{NC}$ into cells, and the luciferase activity was detected using the Dual-Luciferase Assay System. Co-transfection with miR-486 mimics significantly decreased the firefly luciferase activity of Sirt1-3'UTR reporter but not that of the pGL3 reporter $(\mathrm{P}<0.01$; Fig. 5C). Next, we also observed downregulation of Sirt1 in the miR-486 overexpression group by western blot analysis (Fig. 5D). Moreover, the IHC staining indicated that Sirtl expression was downregulated in miR-486 overexpression cell-derived tumor tissues (Fig. 5E). In summary, these findings indicated that Sirt1 is a direct target of miR-486.

Sirtl is increased in liver CSCs and is responsible for maintaining CSC properties. Sirt1 mRNA levels were assessed in tumor spheres and adherent cells. The expression levels of 
A miRecords, RNAhybrid online prediction
Sirt1 3'UTR
5' A UUG UGCA GGUACAGGAA 3'
miR-486 3’ GAGC CC CGU CGAG UCAUGUCCU 5

B
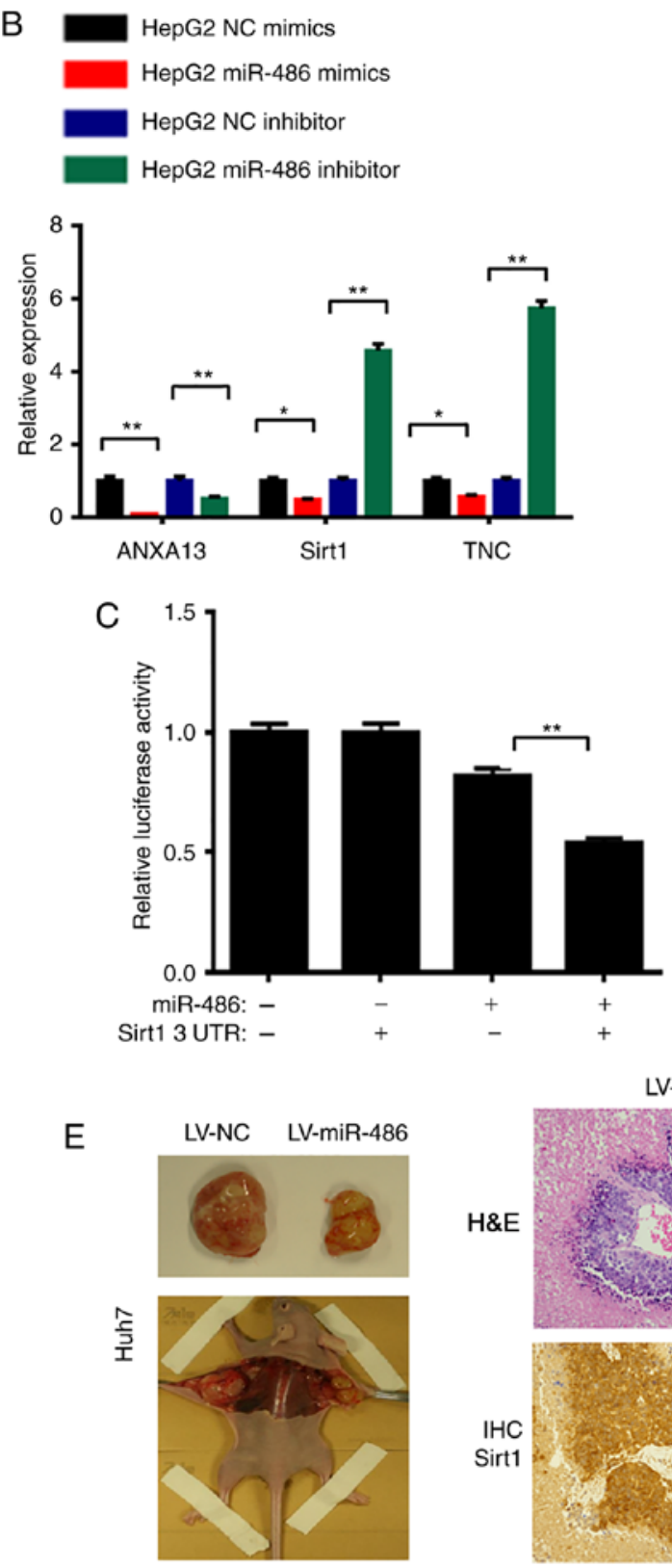

LV-NC

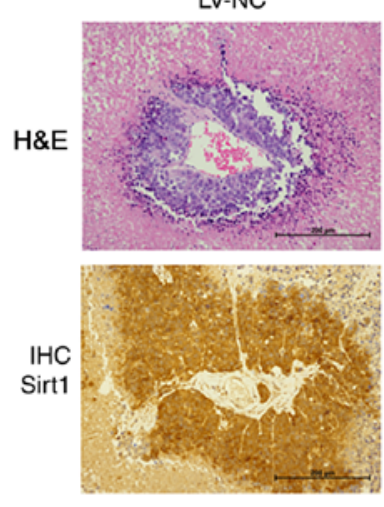

PLC NC mimics

PLC miR-486 mimics

PLC NC inhibitor

PLC miR-486 inhibitor

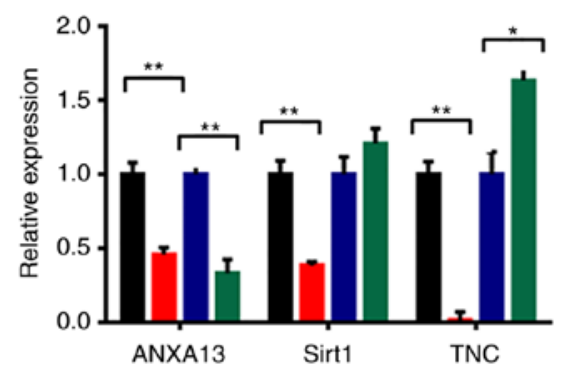

D

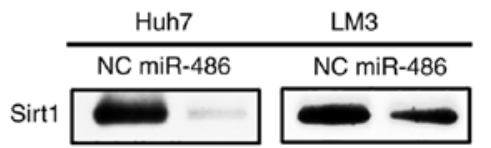

GAPDH

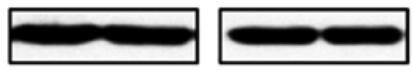

LV-miR-486
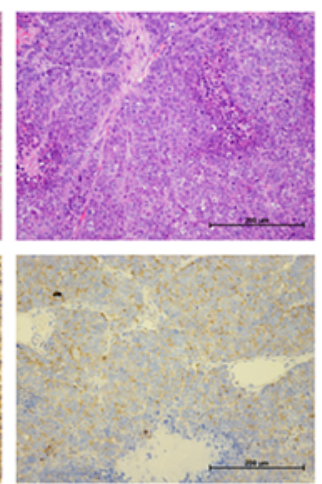

Figure 5. Sirt1 is a direct target of miR-486 in liver CSCs cells. (A) Diagram of the 3'UTR of Sirt1 with putative miR-486-binding sites. (B) qPCR assays were performed to analyze the mRNA expression of ANXA13, Sirt1 and TNC in tumor cells (left panel, HepG2 cells; right panel, PLC cells) transfected with miR-486 mimics or miR-486 inhibitor. Data are displayed as the means \pm SD from at least 3 independent experiments. $\left({ }^{*} \mathrm{P}<0.05,{ }^{* *} \mathrm{P}<0.01\right)$. $(\mathrm{C}) \mathrm{Luciferase}$ reporter assays were used to assess the direct targeting of Sirt1 by miR-486. Multiple groups were compared by a one- or two-way analysis of variance with Tukey's post hoc correction. (D) Western blot analysis of Sirt1 expression in tumor cells transfected with lentivirus miR-486 mimics or control group (NC). (E) IHC staining indicated that Sirt1 expression was downregulated in miR-486-overexpressed cell-derived tumor tissues. CSCs, cancer stem cells; ANXA13, Annexin A13; TNC, tenascin-C; IHC, immunohistochemistry.

Sirt1 were significantly higher in tumor spheres compared with adherent cells $(\mathrm{P}<0.01$; Fig. 6A). This was consistent with our results revealing that miR-486 expression was decreased in tumor spheres and that Sirt1 expression was inversely associated with miR-486 levels. Additionally, increased Sirt1 expression was observed in liver cancer tissues compared with adjacent non-tumor tissues (Fig. 6B).
Then, the expression of Sirt1 in liver cancer cell lines (Fig. 6C) was analyzed. To understand the role of Sirt1 in the maintenance of liver CSC characteristics, we transfected liver cancer cells with Sirt1-shRNA lentivirus, and confirmed that its expression was suppressed by qPCR analyses (Fig. 6D). To determine the biological function of Sirt1 in maintaining CSC-like characteristics, tumor 

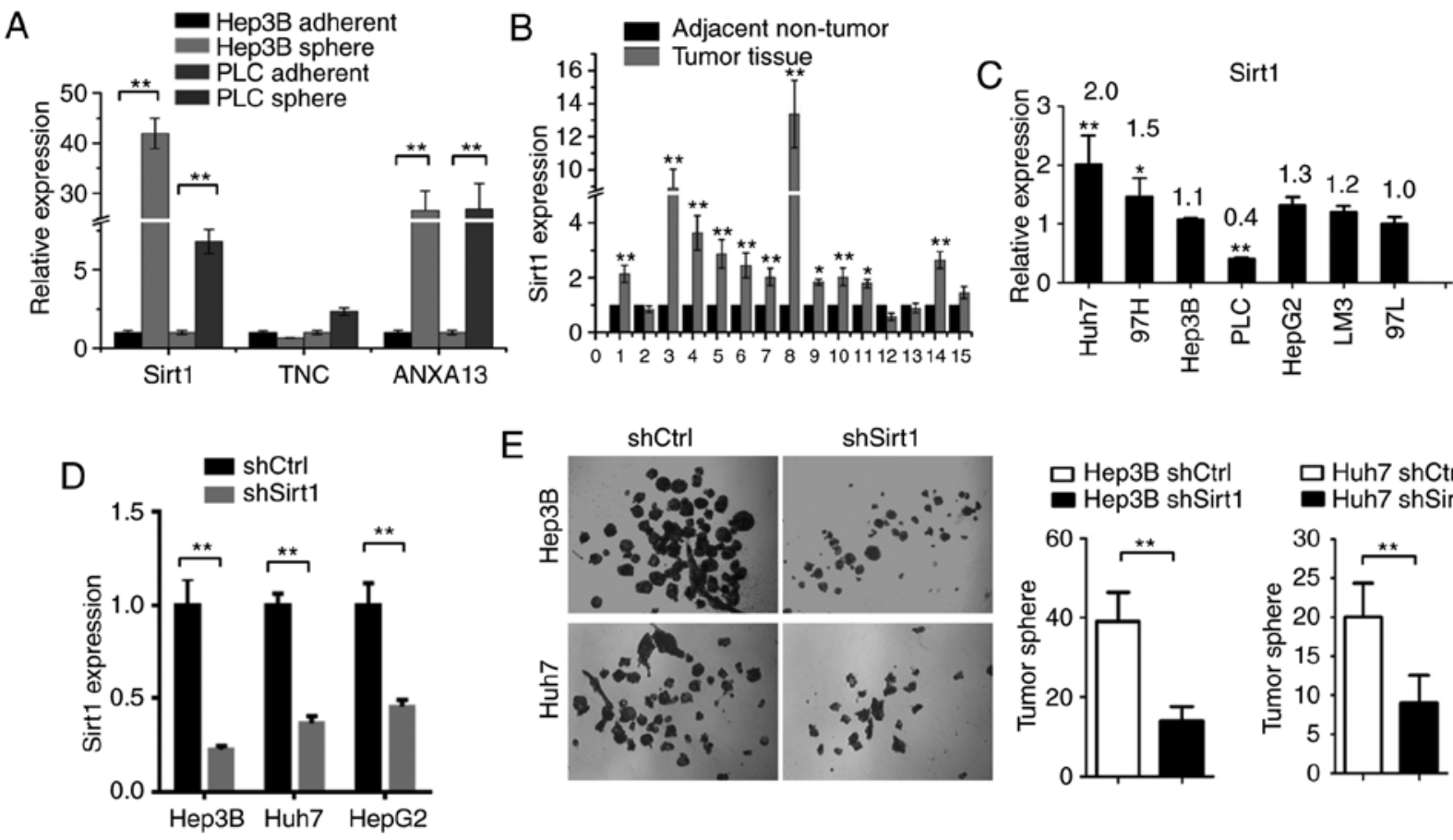

E
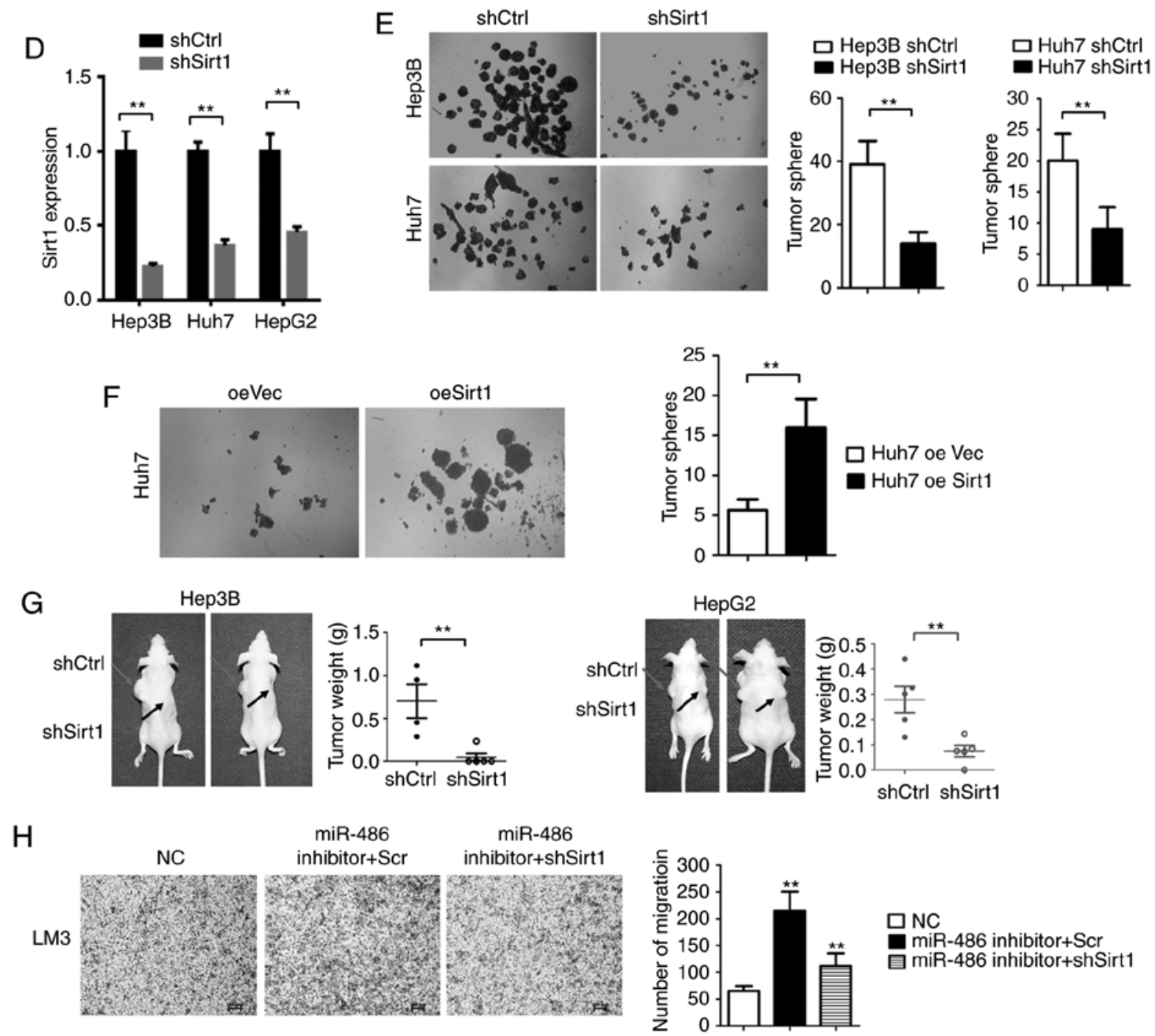

Figure 6. Sirt1 expression is increased in liver CSCs and is responsible for maintaining CSC properties. (A) qPCR assays compared the expression of ANXA13, Sirt1 and TNC in liver tumor spheres and their corresponding adherent cells. (B) qPCR analysis of Sirt1 expression in 16 paired liver cancer and adjacent tissues. (C) qPCR analysis of Sirt1 expression in liver cancer cell lines. (D) qPCR assays demonstrated efficient Sirt1 knockdown in tumor cells via lentivirus transfection of Sirt1 shRNA. (E) Sirt1 knockdown significantly suppressed tumor sphere formation in liver cancer cells. (F) Sirt1 overexpression significantly enhanced tumor sphere formation in Huh7 cells. (G) Knockdown of Sirt1 in liver cancer cells significantly reduced tumor size and tumor weights in nude mice. (H) Sirt1 knockdown reduced the invading capacity of the miR-486 inhibitor-transfected LM3 cells. Data are displayed as the means \pm SD from at least 3 independent experiments. ${ }^{*} \mathrm{P}<0.05,{ }^{* *} \mathrm{P}<0.01$. CSC, cancer stem cells; ANXA13, Annexin A13; TNC, tenascin-C.

sphere assays were performed. As revealed in Fig. 6E, knockdown of Sirtl markedly inhibited tumor sphere formation. Conversely, overexpression of Sirt1 (oeSirt1) was capable of promoting tumor sphere formation (Fig. 6F). To further investigate the biological functions of Sirt1 in liver cancer progression in vivo, HepG2-shSirt1 and HepG2 control cells were subcutaneously injected into nude mice to monitor tumor growth. Tumor weights were reduced in the HepG2-shSirt1 cells as compared with the control group (Fig. 6G). Similar results were obtained with Hep3B-shSirt1 cells (Fig. 6G). Moreover, Sirt1 knockdown reduced the invading capacity of the miR-486 inhibitor-transfected LM3 
cells (Fig. 6H). Collectively, these data indicated that Sirt1 was responsible for the maintenance of self-renewal of liver CSCs and tumorigenesis in vivo.

\section{Discussion}

CSCs or TICs are believed to be the main cause of drug resistance and relapse in liver cancer patients (10). However, few effective interventions have been found for the elimination of CSCs. Liver CSCs have been enriched through a variety of surface markers such as EpCAM, CD13, CD24, $\mathrm{CD} 90$ and CD133. Subgroups of $\mathrm{CD}^{+}{ }^{+}$and $\mathrm{EpCAM}^{+}$play important roles in liver cancer propagation and relapse (12). miRNAs can affect nearly $30 \%$ of human genes by binding to the 3'UTR of target mRNAs. Recent studies have reported that several miRNAs participate in carcinogenesis (33). In the present study, we utilized easy-to-use tumor spheres to enrich liver CSCs and to identify differentially expressed miRNAs between tumor spheres and adherent cells. We mainly investigated the differentially expressed miRNAs that were significantly downregulated in the TCGA database and validated the expression of these miRNAs in tumor spheres and liver CSCs $\left(\mathrm{CD}_{3} 3^{+}\right.$or $\left.\mathrm{EpCAM}^{+}\right)$.

Among these miRNAs, we found that miR-486 was significantly downregulated in tumor spheres, $\mathrm{CD}^{3} 3^{+}$or $\mathrm{EpCAM}^{+}$ liver CSCs. Previous studies have indicated that miR-486 can function as a tumor suppressor in lung (34) and gastric cancer (35). However, it was unknown whether miR-486 regulates the self-renewal properties of liver CSCs. In the present study, it was revealed that miR-486 expression was downregulated in $\mathrm{CD}_{13}{ }^{+}$and $\mathrm{EpCAM}^{+}$liver CSCs compared with the control group. In addition, miR-486 significantly suppressed liver CSC properties, tumorigenesis, chemoresistance and the invasion process of liver cancer cells.

To further clarify the mechanism of miR-486 inhibition of cancer stemness, the TargetScan database and qPCR assays to screen the potential target genes of miR-486 were used. Consequently, it was revealed that Sirt1 was significantly suppressed by miR-486. Moreover, the expression levels of Sirt1 in tumor spheres were markedly higher compared with adherent cells. The luciferase assay revealed that miR-486 significantly decreased the firefly luciferase activity of the Sirt1 3'UTR reporter. Collectively, these data revealed that Sirt1 was a direct target of miR-486. Sirt1 expression has been revealed to be associated with poor prognosis and development of liver cancer in patients (36). The present findings revealed that knockdown of Sirt1 inhibited tumor sphere formation. Conversely, overexpression of Sirt1 significantly promoted tumor sphere formation. Moreover, the present study demonstrated that Sirtl was required for liver cancer tumorigenesis in vivo. Collectively, we found that Sirt1 was responsible for the maintenance of the self-renewal of liver CSCs. Our data was consistent with a study by Liu et al (36), which revealed that Sirtl promoted tumorigenesis by mediating the activation of SOX2. It was revealed in the present results that the expression of both Sirt1 and SOX2 expression was suppressed by miR-486.

In summary, the present study demonstrated that miR-486, which was significantly downregulated in liver CSCs, suppressed liver CSCs by targeting Sirt1. Thus, the
miRNA-486/Sirt1 axis may provide new insights into the mechanism of liver CSCs and may be a useful therapeutic target for liver cancer.

\section{Acknowledgements}

We thank Zeng Fan (Institute of Health Service and Transfusion Medicine, Beijing, China) for technical support. We thank LetPub (www.letpub.com) for providing linguistic assistance during the preparation of this manuscript.

\section{Funding}

The present study was supported by the National Natural Science Foundation of China (nos. 81472341, 81772617, 81773137 and 81502581), the Beijing Municipal Education Commission (no. KM201710005031) and the Guangzhou Health Care and Cooperative Innovation Major Project (no. 201803040005).

\section{Availability of data and materials}

The data sets used during the present study are available from the corresponding author upon reasonable request.

\section{Authors' contributions}

$\mathrm{XY}$ conceived and designed the study, performed the experiments, analyzed the data and wrote the manuscript; XL and QC performed the experiments and wrote the manuscript; $\mathrm{ZW}$ provided the clinical specimens and analyzed the data. GJ, HY, LW, CG, QZ, JX, LH and XN performed the experiments and analyzed the data; $\mathrm{HH}$ constructed the plasmids and analyzed the data; WY and XP initiated the study, organized, designed and wrote the manuscript. All authors read and approved the manuscript and agree to be accountable for all aspects of the research in ensuring that the accuracy or integrity of any part of the work are appropriately investigated and resolved.

\section{Ethics approval and consent to participate}

The Research Ethics Committees of the Academy of Military Medical Sciences, Beijing, China. Written informed consent was obtained from all patients. All animal experiments in the present study were approved by the Ethics Committee of the Academy of Military Medical Sciences.

\section{Patient consent for publication}

Not applicable.

\section{Competing interests}

The authors declare that they have no competing interests.

\section{References}

1. Torre LA, Bray F, Siegel RL, Ferlay J, Lortet-Tieulent J and Jemal A: Global cancer statistics, 2012. CA Cancer J Clin 65: 87-108, 2015.

2. Visvader JE: Cells of origin in cancer. Nature 469: 314-322, 2011. 
3. Easwaran H, Tsai HC and Baylin SB: Cancer epigenetics: Tumor heterogeneity, plasticity of stem-like states, and drug resistance. Mol Cell 54: 716-727, 2014.

4. Kreso A and Dick JE: Evolution of the cancer stem cell model. Cell Stem Cell 14: 275-291, 2014.

5. Liu C, Kelnar K, Liu B, Chen X, Calhoun-Davis T, Li H, Patrawala L, Yan H, Jeter C, Honorio S, et al: The microRNA miR-34a inhibits prostate cancer stem cells and metastasis by directly repressing CD44. Nat Med 17: 211-215, 2011

6. Muthukrishnan SD, Alvarado AG and Kornblum HI: Building bonds: Cancer stem cells depend on their progeny to drive tumor progression. Cell Stem Cell 22: 473-474, 2018.

7. Liu C, Liu L, Chen X, Cheng J, Zhang H, Shen J, Shan J, Xu Y, Yang Z, Lai M and Qian C: Sox9 regulates self-renewal and tumorigenicity by promoting symmetrical cell division of cancer stem cells in hepatocellular carcinoma. Hepatology 64: 117-129, 2016.

8. Zhang L, Sun H, Zhao F, Lu P, Ge C, Li H, Hou H, Yan M, Chen T, Jiang G, et al: BMP4 administration induces differentiation of $\mathrm{CD}_{133^{+}}$hepatic cancer stem cells, blocking their contributions to hepatocellular carcinoma. Cancer Res 72: 4276-4285, 2012.

9. Tang KH, Ma S, Lee TK, Chan YP, Kwan PS, Tong CM, Ng IO, Man K, To KF, Lai PB, et al: CD133+ liver tumor-initiating cells promote tumor angiogenesis, growth, and self-renewal through neurotensin/interleukin-8/CXCL1 signaling. Hepatology 55: 807-820, 2012.

10. Yang ZF, Ho DW, Ng MN, Lau CK, Yu WC, Ngai P, Chu PW, Lam CT, Poon RT and Fan ST: Significance of CD $90^{+}$cancer stem cells in human liver cancer. Cancer Cell 13: 153-166, 2008.

11. Mani SK, Zhang H, Diab A, Pascuzzi PE, Lefrançois L, Fares N, Bancel B, Merle P and Andrisani O: EpCAM-regulated intramembrane proteolysis induces a cancer stem cell-like gene signature in hepatitis B virus-infected hepatocytes. J Hepatol 65 : 888-898, 2016

12. Haraguchi N, Ishii H, Mimori K, Tanaka F, Ohkuma M, Kim HM, Akita H, Takiuchi D, Hatano H, Nagano H, Barnard GF, et al: CD13 is a therapeutic target in human liver cancer stem cells. J Clin Invest 120: 3326-3339, 2010.

13. Christ B, Stock P and Dollinger MM: CD13: Waving the flag for a novel cancer stem cell target. Hepatology 53: 1388-1390, 2011.

14. Kawai T, Yasuchika K, Ishii T, Katayama H, Yoshitoshi EY, Ogiso S, Kita S, Yasuda K, Fukumitsu K, Mizumoto M, et al: Keratin 19, a cancer stem cell marker in human hepatocellular carcinoma. Clin Cancer Res 21: 3081-3091, 2015.

15. Yang W, Wang C, Lin Y, Liu Q, Yu LX, Tang L, Yan HX, Fu J, Chen Y, Zhang HL, et al: $\mathrm{OV}^{+}$tumor-initiating cells contribute to tumor progression and invasion in human hepatocellular carcinoma. J Hepatol 57: 613-620, 2012.

16. Yamashita T and Wang XW: Cancer stem cells in the development of liver cancer. J Clin Invest 123: 1911-1918, 2013.

17. Chiba T, Kita K, Zheng YW, Yokosuka O, Saisho H, Iwama A, Nakauchi $\mathrm{H}$ and Taniguchi $\mathrm{H}$ : Side population purified from hepatocellular carcinoma cells harbors cancer stem cell-like properties. Hepatology 44: 240-251, 2006.

18. Visvader JE and Lindeman GJ: Cancer stem cells in solid tumours: Accumulating evidence and unresolved questions. Nat Rev Cancer 8: 755-768, 2008.

19. Di Leva G, Garofalo M and Croce CM: MicroRNAs in cancer. Annu Rev Pathol 9: 287-314, 2014.

20. Bartel DP: MicroRNAs: Target recognition and regulatory functions. Cell 136: 215-233, 2009.

21. Ladeiro Y, Couchy G, Balabaud C, Bioulac-Sage P, Pelletier L, Rebouissou S and Zucman-Rossi J: MicroRNA profiling in hepatocellular tumors is associated with clinical features and oncogene/tumor suppressor gene mutations. Hepatology 47: 1955-1963, 2008.
22. Borel F, Konstantinova P and Jansen PL: Diagnostic and therapeutic potential of miRNA signatures in patients with hepatocellular carcinoma. J Hepatol 56: 1371-1383, 2012

23. Yan XL, Jia YL, Chen L, Zeng Q, Zhou JN, Fu CJ, Chen HX, Yuan HF, Li ZW, Shi L, et al: Hepatocellular carcinoma-associated mesenchymal stem cells promote hepatocarcinoma progression: Role of the S100A4-miR155-SOCS1-MMP9 axis. Hepatology 57: 2274-2286, 2013.

24. Chai S, Ng KY, Tong M, Lau EY, Lee TK, Chan KW, Yuan YF, Cheung TT, Cheung ST, Wang XQ, et al: Octamer $4 / \mathrm{micro}-$ RNA-1246 signaling axis drives Wnt/ $\beta$-catenin activation in liver cancer stem cells. Hepatology 64: 2062-2076, 2016.

25. Li Y, Tang ZY, Ye SL, Liu YK, Chen J, Xue Q, Chen J, Gao DM and Bao WH: Establishment of cell clones with different metastatic potential from the metastatic hepatocellular carcinoma cell line MHCC97. World J Gastroenterol 7: 630-636, 2001.

26. Livak KJ and Schmittgen TD: Analysis of relative gene expression data using real-time quantitative PCR and the 2(-Delta Delta C(T)) Method. Methods 25: 402-408, 2001.

27. Yan XL, Fu CJ, Chen L, Qin JH, Zeng Q, Yuan HF, Nan X, Chen HX, Zhou JN, Lin YL, et al: Mesenchymal stem cells from primary breast cancer tissue promote cancer proliferation and enhance mammosphere formation partially via EGF/EGFR/Akt pathway. Breast Cancer Res Treat 132: 153-164, 2012.

28. Miao X, Yan X, Qu D, Li D, Tao FF and Sun Z: Red emissive sulfur, nitrogen codoped carbon dots and their application in ion detection and theraonostics. ACS Appl Mater Interfaces 9: 18549-18556, 2017.

29. Zhou JN, Zeng Q, Wang HY, Zhang B, Li ST, Nan X, Cao N, Fu CJ, Yan X, Jia YL, et al: MicroRNA-125b attenuates epithelial-mesenchymal transitions and targets stem-like liver cancer cells through small mothers against decapentaplegic 2 and 4. Hepatology 62: 801-815, 2015.

30. Yan X, Zhang D, Wu W, Wu S, Qian J, Hao Y, Yan F, Zhu P, Wu J, Huang G, et al: Mesenchymal stem cells promote hepatocarcinogenesis via lncRNA-MUF interaction with ANXA2 and miR-34a. Cancer Res 77: 6704-6716, 2017.

31. Li Y, Tian B, Yang J, Zhao L, Wu X, Ye SL, Liu YK and Tang ZY: Stepwise metastatic human hepatocellular carcinoma cell model system with multiple metastatic potentials established through consecutive in vivo selection and studies on metastatic characteristics. J Cancer Res Clin Oncol 130: 460-468, 2004

32. Volinia S, Calin GA, Liu CG, Ambs S, Cimmino A, Petrocca F, Visone R, Iorio M, Roldo C, Ferracin M, et al: A microRNA expression signature of human solid tumors defines cancer gene targets. Proc Natl Acad Sci USA 103: 2257-2261, 2006.

33. Gramantieri L, Ferracin M, Fornari F, Veronese A, Sabbioni S, Liu CG, Calin GA, Giovannini C, Ferrazzi E, Grazi GL, et al: Cyclin G1 is a target of miR-122a, a microRNA frequently downregulated in human hepatocellular carcinoma. Cancer Res 67: 6092-6099, 2007.

34. Wang J, Tian X, Han R, Zhang X, Wang X, Shen H, Xue L, Liu Y, Yan X, Shen J, et al: Downregulation of miR-486-5p contributes to tumor progression and metastasis by targeting protumorigenic ARHGAP5 in lung cancer. Oncogene 33: 1181-1189, 2014.

35. Oh HK, Tan AL, Das K, Ooi CH, Deng NT, Tan IB, Beillard E, Lee J, Ramnarayanan K, Rha SY, et al: Genomic loss of miR-486 regulates tumor progression and the OLFM4 antiapoptotic factor in gastric cancer. Clin Cancer Res 17: 2657-2667, 2011.

36. Liu L, Liu C, Zhang Q, Shen J, Zhang H, Shan J, Duan G, Guo D, Chen X, Cheng J, et al: SIRT1-mediated transcriptional regulation of SOX2 is important for self-renewal of liver cancer stem cells. Hepatology 64: 814-827, 2016. 